\title{
Joint assessment out of hours: assessing the benefits for patients and junior doctors
}

\author{
Laura Boyd, ${ }^{1}$ Colin Crawford, ${ }^{1}$ Eugene Wong ${ }^{2}$
}

The Psychiatrist (2013), 37, 272-275, doi: 10.1192/pb.bp.112.041210

${ }^{1}$ NHS Forth Valley, Scotland, UK; ${ }^{2} \mathrm{NHS}$ Greater Glasgow and Clyde, Scotland, UK

Correspondence to Laura Boyd (lauraboyd1@nhs.net)

First received 15 Aug 2012, final revision 13 Mar 2013, accepted 14 Mar 2013

\begin{abstract}
Aims and method We examined the impact of implementing a new Acute Mental Health Emergency Assessment Protocol (AMHEAP) on joint psychiatric assessments out of hours within Forth Valley, Scotland, over the course of 4 calendar months. The protocol states that assessments should be carried out by a junior doctor and a registered, qualified mental health nurse. The impact measures were taken as admission rates and experience of the doctor in training.
\end{abstract}

Results In the 4 months that were examined (1 June-30 September 2011), 79.5\% of out-of-hours emergency assessments were performed jointly. Admission rates were significantly decreased $(P<0.001)$ compared with a similar period in 2008 , before the AMHEAP protocol was developed. Most junior doctors valued the experience of joint assessment.

Clinical implications Joint assessment can enhance patient experience, reduce hospital admission, and provide a learning opportunity for junior doctors in emergency psychiatric assessments. However, it represents a move away from the doctor as sole decision maker.

\section{Declaration of interest None.}

Since the introduction of the European Working Time Directive in August 2004, there has been much debate about its effect on the experience of the core trainee, be it in psychiatry or elsewhere. ${ }^{1}$ It has been shown that with the establishment of a new generation of acute psychiatric subspecialties, such as crisis and home treatment teams, junior doctors working in mental health (foundation year doctors, general practice trainees and core psychiatry trainees) are carrying out fewer emergency assessments, with those which are still performed largely occurring in an on-call capacity where opportunity for teaching is limited. ${ }^{2}$ This reduces the availability of assessment experience and the frequency with which management plans are constructed by the core trainee. Additionally, certain conditions are more likely to present for assessment out of hours, for example cases of self-harm which are often referred to an inexperienced junior doctor for assessment, often without guidance or supervision from a senior colleague. ${ }^{2-4}$ It is likely that this will have an impact on acquisition of core decision-making skills as the core trainee progresses to higher-level training and post-certificate of completion of training (CCT).

\section{Study setting}

NHS Forth Valley covers three local council authorities: Clackmannanshire, Falkirk and Stirling, with a population of almost 300000 in a semi-rural setting. ${ }^{5}$ In 2005, the intensive home treatment team (IHTT) was launched in the region to offer a less restrictive alternative to hospital admission. During their operational hours they act as gatekeepers, triaging and performing emergency assessments. The team operates from 9am to 9pm Monday to Friday, and 10am to $6 \mathrm{pm}$ Saturday and Sunday. Outwith these hours, or where capacity is reached, the role is passed to the junior doctor on call.

In the Best Practice Statement released by NHS Quality Improvement Scotland in 2009 it was suggested that where possible, mental health assessments should be based on a multidisciplinary approach. ${ }^{6}$ It is further stated that all options should be considered, together with exploration of alternatives to hospital admission. This would require knowledge of local resources, which perhaps doctors new to a rotation are not afforded. NHS Forth Valley introduced the Acute Mental Health Emergency Assessment Protocol (AMHEAP) in April 2011. ${ }^{7}$ The aim of the new protocol was to support junior doctors, who traditionally completed emergency assessments on their own, in an attempt to achieve a comprehensive assessment and the most suitable, least restrictive patient outcome. This too may offer significant benefit for juniors with little or no mental health experience faced with the demands of being on call. For example, August 2011 saw the highest number of out-ofhours referrals over a 1-month period coinciding with the traditional changeover period.

Perhaps now it is time to enhance the learning opportunities of those less frequent emergency assessments. 
The Royal College of Psychiatrists points out that where a multitude of alternative services - liaison, crisis, IHTT, 24hour community psychiatric nurses - exist, trainees are largely excluded from gaining experience in emergency assessments. Therefore alternative arrangements should be made ensuring this valuable experience is obtained under supervision. $^{8}$

Our local (AMHEAP) protocol advocates that whenever possible, emergency assessments should be carried out by the junior doctor and another member of the multidisciplinary team. ${ }^{7}$ In the first instance this should be the junior doctor and a nominated registered mental health nurse who has successfully completed the degree-level mental health assessment course, which was developed in partnership by NHS Forth Valley and the Faculty of Nursing and Midwifery at the University of Stirling.

\section{Aim}

Our aim was to evaluate and discuss the impact of this local change of procedure and introduction of nurses trained in assessment on clinical outcome and the training of junior doctors in psychiatry. It is hoped we will see a more positive training experience as well as an increase in positive risktaking. Risk is an everyday experience and within mental health it is something we are constantly addressing, although this is often hindered by fear of adverse outcomes, for example self-harm or suicide. Positive risk-taking requires consideration of potential benefits and consequences of a proposed outcome, thus allowing a management plan to be developed which reflects an individual's potential, incorporates available resources and ultimately minimises harm. ${ }^{9}$

\section{Method}

At the introduction of the AMHEAP, junior doctors were asked to complete an evaluation form for each out-of-hours assessment undertaken. This form gathered the following information: age of the person assessed, whether the assessment was joint or solo, if carried out solo a reason for the same, and outcome of the assessment. For the purpose of this audit, forms were examined over 4 months extending from 1 June to 30 September 2011. Data were analysed with SPSS version 20.0.0 on Windows. We used Pearson's $\chi^{2}$ to identify any significant associations between admission rates and the introduction of the AMHEAP.

Additionally, individual semi-structured interviews were undertaken with 9 of 10 junior doctors participating in the on-call rota over the study period to gather qualitative information regarding the implementation of the protocol (1 doctor was unavailable to comment due to leave). The topic guides were simple and non-directive, gathering opinions on the process itself in addition to any positive or negative comments. These were audio-recorded and later transcribed.

\section{Results}

Over the 4 months of the study, a total of 176 out-of-hours assessments were performed by junior medical staff. This averages 1.4 assessments per night and is in keeping with an earlier study within Forth Valley where the number totalled 168 assessments. $^{2}$ Overall, 122 evaluation forms were completed, giving a response rate of $69.3 \%$.

In total, 97 assessments (79.5\%) were recorded as undertaken by a doctor and a trained assessment nurse. Previously these would have been completed by the doctor in training only.

In a comparative 4-month period analysed in an earlier study in Forth Valley, ${ }^{2}$ we saw $56 \%$ of those assessed by the junior doctor alone admitted to an acute psychiatric ward. The rate of admission following out-of-hours assessment since the introduction of the AMHEAP protocol was $26 \%$ (32/122) based on data from completed evaluation forms. Taking into account the data missed by this method, 49 admissions were generated from the total 176 assessments (28\%). This is summarised in Table 1.

A $2 \times 2 \chi^{2}$-test was carried out using SPSS version 20.0.0 to discover whether there was a significant relationship between the number of referred individuals admitted to the mental health unit following traditional assessment by junior doctors alone, and after AMHEAP was introduced promoting joint assessment.

The $\chi^{2}$ value of 29.10 (two-tailed) had an associated $P<0.0001$, d.f. $=1$, showing that such an association (the change in number of patients admitted) is extremely unlikely to have arisen as a result of sampling error. Cramer's $V$ was 0.29 , thus around $8.41 \%$ of the variation in frequencies of admissions can be explained by AMHEAP.

Therefore, we can conclude that admission rates have significantly decreased since making joint assessments standard practice.

Although widely promoted, joint assessment has not always proved globally feasible due to clinical activity within the mental health unit. In this situation the doctor will perform the assessment independently. During our 4month study there was no statistical significance seen when comparing the outcome of joint assessments with those performed by the junior doctor alone. We could surmise that in addition to leading to a decrease in admission rates, the contribution of nursing staff to emergency assessments by junior doctors has also resulted in increased confidence in assessing patients alone when the gold standard of joint assessment cannot be facilitated.

In general, all of the junior doctors interviewed made positive comments after having the opportunity to participate in joint assessments. A selection of anonymised responses is given in Box 1 .

\begin{tabular}{|c|c|c|c|c|}
\hline \multirow{3}{*}{$\begin{array}{l}\text { Table } 1 \\
\text { Year } \\
\end{array}$} & \multicolumn{3}{|c|}{$\begin{array}{l}\text { Outcome of out-of-hours emergency } \\
\text { assessments, before and after AMHEAP } \\
\text { implementation }\end{array}$} & \multirow[b]{3}{*}{ Total } \\
\hline & & \multicolumn{2}{|c|}{ Referred individuals $n(\%)$} & \\
\hline & & Admitted & Not admitted & \\
\hline \multicolumn{2}{|c|}{ 2008, pre-AMHEAP ${ }^{a}$} & $95(56.5)$ & $73(43.5)$ & 168 \\
\hline \multicolumn{2}{|c|}{ 2011, post-AMHEAP } & $49(27.8)$ & $127(72.2)$ & 176 \\
\hline
\end{tabular}

AMHEAP, Acute Mental Health Emergency Assessment Protocol. a. Data from Waddell \& Crawford. $^{2}$ 


\section{Box 1 Junior doctors' comments on joint assessments}

'They've [assessment nurses] got a lot of clinical experience ... a really good knowledge of local services and how things work within the unit. They can help you come up with a reasonable plan $[\ldots]$ and either confirm your thoughts or offer an alternative.'

'I started on nights, was new and it was good to have an assessor to ask and get tips on technique, etc, bounce ideas off.'

'Very positive [experience], safer [ . . . W Whatever is lacking should be made available to provide constant nurse cover.'

'Helpful, particularly when setting boundaries for patients. De-escalation was particularly effective.'

'[Assessing alone was] more stressful, potential for allegations, etc. Might tend to admit more as it is the safe thing to do.'

However, not all comments were as positive:

'On occasion I found it very difficult and that was only if I felt my rapport with the patient was being adversely affected by there being another person there [ . . . ] if you've got someone with you who is chipping in asking other questions it can divert your focus.'

'There is a risk people get worked up and anxious with more than one person.'

\section{Discussion}

The trainees' exposure to emergency psychiatric assessments is diminishing. The majority now occur out of hours, particularly overnight when there is typically no resident senior doctor or consultant to ask for advice or utilise the opportunity for teaching. Understandably, junior doctors can lack the confidence in their abilities when faced with the new challenges that psychiatry brings. There is perhaps no other specialty quite like it in terms of deciding which individuals require emergency admission. The junior doctor out of hours may not have the experience in assessing risk, or simply fear their good intentions going wrong. Additionally, in some cases admission may be against the long-term interests of the patient. ${ }^{4}$ Here we can incorporate the development of positive risk-taking, not in the context of denying a service but building on strengths and abilities of the patient and prior knowledge of successful and unsuccessful interventions. So, can the registered nurse trained in mental health assessment promote a better experience for both patient and doctor in training?

Since the implementation of the AMHEAP we have observed a statistically significant reduction in the number of admissions generated from out-of-hours emergency assessment. These data provide a 4-month snapshot. Taking the annual departmental figures we have observed a $10 \%$ reduction in out-of-hours admissions, whereas the number of assessments has remained comparable. This would suggest increased utilisation of alternative assessment outcomes and positive risk-taking. It should be noted that there has been no increase in adverse clinical events recorded as a consequence. NHS Forth Valley are continuing to promote the use of AMHEAP and extending it to the older people's service. This is facilitated by enrolment of additional registered mental health nurses to the mental health assessment course, as well as ongoing support for those members of staff who have already achieved competency in this.

\section{Limitations}

There are of course limitations to our findings. Acute mental health services have relocated to a new general hospital site at Forth Valley Royal Hospital, Larbert, since the 2008 data were collected. Prior to this the junior doctor would cover two acute hospital sites out of hours. The population served is unchanged and although relocation of the hospital may have been a contributory factor, absolute numbers of referrals are remarkably similar over the two 4-month periods sampled in our and Waddell \& Crawford's ${ }^{4}$ study. The comparison data in this audit were of an overlapping but not identical 4 months of the year. Additionally, the earlier cohort of junior doctors have moved on and have been replaced by an unmatched group in terms of training grade.

Regarding training, the presence of an experienced member of nursing staff can offer junior medical staff a learning opportunity, allowing them to develop and refine not only their assessment style but an ability to prepare a comprehensive biopsychosocial formulation and management plan, which many may have not had to consider in previous medical posts.

Having the opportunity to perform assessments with a range of staff improves the training experience. However, there may also be a risk attached. Although the traditional role of the nurse is expanding, nurses differ in both undergraduate and postgraduate training. Not only that, junior doctors and nurses differ in their perceived level of responsibility.

There is a danger that those doctors starting out in training who never experience assessing on their own may not acquire essential decision-making skills. They may not learn to take professional responsibility or gain the confidence to make difficult clinical decisions, when ultimately, "The aim of training is to provide a doctor with an educational programme, which enables him or her to practice independently in psychiatry, whether as a consultant or as a senior member of an academic department' (p. 42). ${ }^{8}$

The conundrum of whether joint assessment is a junior doctor's friend remains.

\section{About the authors}

Laura Boyd is a CT3 in Psychiatry, and Colin Crawford is a Consultant Psychiatrist, both within NHS Forth Valley, Scotland. Eugene Wong is an ST5 in Psychiatry, now working in NHS Greater Glasgow and Clyde, Scotland. 


\section{References}

1 McLernon C, Coccia F, Patel AG. Adherence to the European Working Time Directive and its influence on clinical experience. Psychiatr Bull 2007; 31: 295-6.

2 Waddell L, Crawford C. Junior doctors are performing fewer emergency assessments - a cause for concern. Psychiatrist 2010; 34: 268-70.

3 Dunn J, Fernando R. Psychiatric presentations to an accident and emergency department. Psychiatr Bull 1989; 13: 672-4.

4 Royal College of Psychiatrists. Self-Harm, Suicide and Risk: A Summary (Position Statement PS3/2010). Royal College of Psychiatrists, 2010.

5 General Register Office for Scotland. National Records of Scotland: Mid2011 Population Estimates. National Records of Scotland, 2012.
6 NHS Quality Improvement Scotland. Admissions to Adult Mental Health Inpatient Services: Best Practice Statement. NHS Quality Improvement Scotland, 2009

7 NHS Forth Valley. Adult Mental Health Emergency Assessments. Adult Mental Health Clinical Management Team, 2011.

8 Royal College of Psychiatrists. Specialist Training in Psychiatry: A Comprehensive Guide to Training and Assessment in the UK for Trainees and Local Educational Providers (Occasional Paper OP69). Royal College of Psychiatrists, 2010.

9 Morgan S. Positive Risk-Taking: An Idea Whose Time Has Come. Practice Based Evidence, 2004 